\title{
Surface Electronic Structure of GaAs(110) Studied by $\quad$ DE92 012211 Auger Photoelectron Coincidence Spectroscopy
}

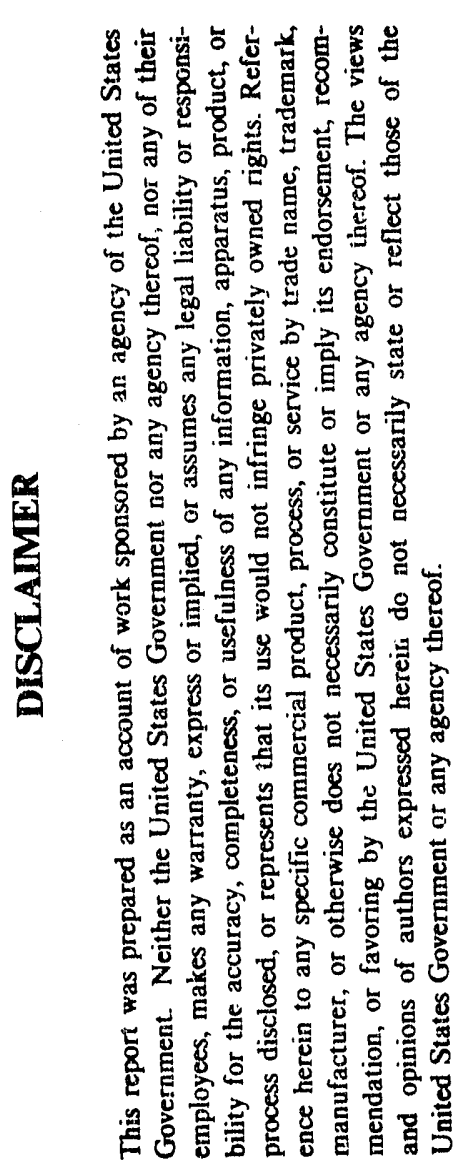

\author{
R.A. Bartynski \\ Department of Physics and Astronomy and \\ The Laboratory for Surface Modification \\ Futgers University, Piscataway NT 08855-0849 \\ E. Jensen \\ Department of Physics, \\ Brandeis Uninersity, Waltham, MA 02254 \\ K. Garrison \\ Department of Physics and Astronomy and \\ - The Laboratory for Surface Modification \\ Rutgers University, Piscataway NT 08855-0849 \\ S.L. Hulbert \\ National Synchrotron Light Source, \\ Brookhaven National Laboratory, Upton, NY 11973 \\ M. Weinert \\ Department of Physics, \\ Brookhaven National Laboratory, Upton, NY 11973
}

We have used Auger photoelectron coincidence spectroscopy to study the $\mathrm{M}_{4,5} \mathrm{VV}$ Auger spectra of GaAs(110). Using this technique, the Ga and As spectra can be separated and studied independently. The lineshape of the $A s-M_{4,5} \mathrm{VV}$ measured in coincidence with the As $3 \mathrm{~d}$ photoemission line differs significantity from the conventional Auger spectrum. We attribute this to the surface electronic properties of the system. In addition, we have found that the sscomponent of the As spectrum is more intense than expected based on calculations using atomic matrix elements. The Ga-M4,5VV spectrum, of which only the pp-component is observed, agrees well with that expected from an independent electron model. A first principles electronic structure calculation of a 5-layer GaAs(110) slab has been performed to aid in the interpretation of the Auger spectra. 


\section{Introduction}

Auger electron spectroscopy, though typically used as a surface characterization technique, can be employed as a probe of electronic structure. ${ }^{1-10}$ This is because the line shape of the Auger transitions which involve valence electrons contains information about the electronic configuration at the site of the core hole. Thus Auger spectroscopy is a potentially powerful technique for studying the local electronic properties of inhomogeneous systems such as compound semiconductors or surfaces. However, there are several factors which must be considered before an analysis of Auger spectral lineshapes in terms of the valence band density of states (DOS) is undertaken. It is well known that matrix elements can influence the Auger line shape. $1,2,5-10$ Typically these effects can be accounted for by separately weighting the contribution of each angular momentum component of the DOS to the Auger spectrum In addition, Auger spectroscopy always involves the presence of a core hole in the initial state and, for all but core-valence-valence (CVV) transitions, a core hole exists in the final state. The potential of the core hole may significantly modify the electronic structure so that the Auger spectrum does not reflect the ground state properties of the system. Finally, in narrow band systems, electron correlations in the two-hole final state may strongly influence the spectral line shape. $2-4$

These concerns can be turned in to advantages, however, since deviations of the Auger lineshape from that predicted by the local DOS contain information about the excitation properties of the system. In this paper we describe an Auger photoelectron coincidence $2-4,8,11,12$ (APECS) sludy of the CVV Auger pectra of the GaAs(110) surface. In APECS, the Auger spectrum associated with the decay of a specific core hole is obtained in coincidence with photoelectrons created in the excitation of that core hole. This additional level of discrimination removes from the spectrum emission originating from other transitions. Thus, overlapping spectral fcatures can be separated for independent study, and inelastic electron background is strongly suppressed. - We have used this feature of APECS to separate the overlapping Ga- and As-M4,5V Auger spectra of GaAs(110) by measuring them in coincidence with their respective $3 \mathrm{~d}$ core level lines. To our knowledge, this is the first direct measurement of the low energy s-derived part of this As Auger line. A further advantage of this technique is that for true coincidence events, both the Auger electron and the photoelectron escape from the solid. Tnis makes coincidence Auger spectra about a factor of two more surface sensitive ${ }^{2}$ than conventional Auger spectra. Thus differences between the conventional Auger lineshape and the coincidence Auger lineshape may be related to the surface properties of the system. We have found that the As Auger lineshape measured in coincidence with the As $3 d$ core level is significantly different from the conventional photon excited Auger spectrum. A comparison of 
the experimental spectra to calculated lineshape indicates that the most likely origin of this discrepancy is the excitation properties of the surface.

In the next section, we describe our experimental configuration and the calculational techniques. In Sect III we present our experimental and theoretical results and in Sec. IV reconcile the differences. Finally, we summarize our findings and conclusions in Sec. V.

\section{Experimental and Theoretical Considerations}

The principles behind APECS and its experimental implementation have been presented elsewhere $2,-4,8,11,12$ and will not be discussed in detail here. Rather, we briefly describe the aspects of APECS that are relevant to this work. An ultra high vacuum (UHV) experimental chamber contains two cylindrical mirror analyzers ${ }^{13}$ (CMA's) focussed on a sample which is illuminated by monochromatized synchrotron radiation. All of the dati reported here were taken on the U12 TCM beamline ${ }^{14}$ of the VUV-ring at the National Synchrotron Light Source. The photon energy was $90 \mathrm{eV}$ and the system had a combined energy resolution (electron+photon) of about $1 \mathrm{eV}$. To acquire a coincidence spectrum, one CMA was held at a fixed kinetic energy in the core photoelectron spectrum while the other CMA swept through the associated Auger spectrum. If electrons were detected in both analyzers within 20 ns of each other, a coincidence event was recorded. All other events were rejected. Wilh finite count rates and a finite time window, there exists the possibility of counting accidental coincidence events caused by electrons from two unrelated excitations entering our analyzers simultaneously. This accidental rate has been subtracted ${ }^{12}$ from the measured coincidence spectrum in a point-by-point fashion leaving only the true coincidence (hereafter simply referred to as coincidence) spectrum. During acquisition of a coincidence spectrum, a conventional (hereafter called singles) Auger spectrum was also recorded.

The sample was a $\mathrm{Zn}$-doped $\left(\mathrm{n} \mathrm{Zn}=10^{18} \mathrm{~cm}^{-3}\right.$ ) GaAs single crystal oriented in the (110) direction. Prior to insertion into the vacuum chamber, the sample was mechanically polished and chemically etched in a $0.1 \% \mathrm{Br} /$ Methanol solution which yielded a mirror-like finish. Upon insertion into the vacuum chamber, the surface was cleaned by repeated cycles of Ar ion bombardment and subsequent annealing until no detectable signal from any elements other than $\mathrm{Ga}$ and $A s$ was present in electron excited Auger spectra and a sharp (1x1) LEED pattern was observed. Auger peak height ratius were consistent with a stoichiometric surface. As has been observed in other studies, ${ }^{15}$ our photoemission showed excellent agreement with published work from the cleaved CaAs(110) surface. ${ }^{16}$ The pressure in the chamber was in the mid $10^{-11}$ 
torr range during the course of the experirrents and the sample showed no signs of contamination during data acquisition.

To aid in the interpretition of the spectroscopic data, a first principles electronic structure calculation was performed for a 5 layer $\mathrm{GaAs}(110)$ slab within a fully linearized augmented plane wave (FLAPW) framework. ${ }^{17}$ The assumed geometry for this heteropolar surface had he As atoms exhibiting an outward relaxation and the $\mathrm{Ga}$ atoms relaxed inward. Atomic coordinates where those determined by Qian and co-workers ${ }^{18}$ to be the minimum energy configuration which agree well with experimental results. The density of states was obtained by sampling $24 \mathrm{k}$-points within the irreducible wedge of the Brillouin zone, and integrating within an atomic sphere of radius 2.2 atomic units.

\section{Results}

A survey scan of the photoemission spectrum from GaAs(110) at $\hbar \omega=90 \mathrm{eV}$ is presented in Fig. 1. As scaled, the solid curve clearly displays the Ga- and As-3d core levels at kinetic energies of $66 \mathrm{eV}$ and $442 \mathrm{eV}$, respectively. Overlayed on this figure are rescaled plots of the valence band region between 70 and $90 \mathrm{eV}$ kinetic energy, and the low kinetic energy Auger region. The As- $\mathrm{M}_{4,5} \mathrm{VV}$ Auger spectrum can be observed in the singles scan after multiplication by a factor of five. Another weak feature occurs in the solid curve near $8 \mathrm{eV}$. This is the Ga M4,5VV Auger emission which overlaps the low energy part of the primary As $M_{4,5 V}$ spectrum. The interpretation of these features is based on conservation of energy arguments assuming hole-hole interaction is weak. Our assignments are in agreement with the previous measurements of Svenssen et al. ${ }^{19}$ Separation of the Ga and As signals as has been done here is essentially impossible by conventional spectroscopic techniques at energies where both the Ga- and As-3d core levels have been excited.

Figure 2 shows the As $M_{4,5} \mathrm{VV}$. Auger spectrum obtained in coincidence with the As $3 d$ core level. To acquire this spectrum, the fixed analyzer was set at a kinetic energy of $44.2 \mathrm{eV}$ while the scanning analyzer swept the kinetic energy range of $12-37 \mathrm{eV}$. The open circles in Fig. 2(a) are the coincidence data and the solid line represents a digital smooth to these points. Several differences between this spectrum and the singles spectrum of Fig. 1 are immediately apparent. Most striking is the reduction of the inelastic electron background and the elimination of any emission above threshold. This is because electrons not associated with the decay of an As-3d core hole are rejected by the coincidence technique. The spectrum exhibits three main features: a peak at $29 \mathrm{eV}$, a second smaller peak at $20 \mathrm{eV}$ and a third weak feature at $15 \mathrm{eV}$. Despite the additional discrimination against secondary electron emission, there is 
still a contribution to the spectrum arising from Auger electrons which have suffered inelastic losses before escaping the crystal. To better analyze the primary features in the coincidence spectrum, we approximate this background by a smooth monotonic polynomial function shown as the dashed curve in Fig.2(a). The detailed form of this featureless background does not influence the conclusions made below.

After subtracting the inelastic contribution from the coincidence data, we arrive at the spectrum presented in Fig. 2(b). The solid line in this figure is the simultaneously acquired singles spectrum after subtraction of a similar background function. The singles and coincidence data were scaled so that the low kinetic energy features in both spectra have approximately the same intensity. Although the overall structure of these spectra is quite similar, the high energy feature in the singles data is much larger than the equivalent feature in the coincidence spectrum. In addition, the high energy peak is narrower in the coincidence spectrum. Putting the caveats ${ }^{1,2,5-10}$ outlined in the Introduction aside for the moment, the lineshape of the CVV Auger spectrum can be approximated by the self-convolution of the local density of states (LDOS) at the site of the core ho: $9.1,2,5-10$ To this end, we employ the results of the electronic structure calculation. The LDOS at the As site for each unique layer are presented in Fig. 3(a). A comparison of these curves shows that at the surface, the LDOS of pcharacter is narrower and its center of gravity closer to the Fermi level than it is for the subsurface layers. For the As s-derived levels, the sub-surface DOS is broadened while the surface layer is peaked at the low binding energy edge of the s-manifold. These results are consistent with earlier calculations $7,20,21$ of the GaAs(110) electronic structure. The selfconvolution of the LDOS (SCDOS) of each layer is shown in Fig 3(b). The self-folds of the pand s- derived states, as well as the p-folded with the s- are labeled pp, ss and sp, respectively, and have been weighted by the ratio pp:sp:ss of $1.0: 0.3: 0.03$ as predicted by atomic calculations of the respective Auger matrix elements. ${ }^{22}$ The sum of these weighted selffolds is shown as the solid line for each layer. Here we see that the narrower surface p-LDOS manifests itself in a narrower SCDOS whose centroid is closer to threshold than for the deeper layers. From conservation of energy, and in agreement with the results of Svenssen et al., ${ }^{19}$ we can assign the large peak near $29 \mathrm{eV}$ in the Auger spectra of Fig. 2 as the pp-channel, while the peaks at $20 \mathrm{eV}$ and $15 \mathrm{eV}$ are primarily sp-derived. Referring back to Fig 2(b), we see that the data indicate an enhancement of the sp-channel relative to the pp-channel in the coincidence spectrum as compared to the singles spectrum.

Coincidence Auger spectra obtained in the $5-13 \mathrm{eV}$ kinetic energy range are shown in Fig. 4 The spectrum given by the circles in Fig 4(a) was acquired in coincidence with the Ga $3 d$ core level at a kinetic energy of $66 \mathrm{eV}$. Similar to the spectrum in Fig. 2(a), a well defined threshold exists, near $12 \mathrm{eV}$ in this case, above which no emission is observed. Below this 
energy, a single broad feature occurs which, e identify as the Ga M4,5V Auger transition. We assume a simple linear background for the inelastic contribution to this spectrum given by the dashed line in this figure. Upon subtracting this background, we arrive at the spectrum given in the lower half of this panel. This spectrum exhibits a single broad maximum at about $9 \mathrm{eV}$ and, with our current statistics, no structure within this peak is apparent. Figure 4 (b) shows the same kinetic energy region obtained in coincidence with the As $3 d$ core level. This spectrum represents an extension of Fig. 2(a) to lower kinetic energy and hence emission throughout the spectrum is expected. There is, however, a single weak feature near $8 \mathrm{eV}$. If a simple linear background is removed from this spectrum the feature is more evident. We identify this peak as the ss-portion of the As $M_{4}, 5 \mathrm{VV}$ Auger spectrum and note that it occurs about $2 \mathrm{eV}$ lower than expected from a noninteracting electron model. Since this emission and the $\mathrm{Ga} \mathrm{M4,5VV}$ spectrum overlap in energy, we know of no other technique which can isolate this As feature as we have done here.

\section{Discussion}

Perhaps the most studied CVV Auger lineshape has been $\mathrm{L}_{2,3} \mathrm{VV}$ transition of $\mathrm{Si}$. In the late 1970 's, Feibelman and co-workers ${ }^{6,7}$ pointed out that for wide band materials where the density of states is composed of more than one angular momentum component, matrix element effects will change the relative contribution of each component to the CVV Auger spectrum. By calculating the relevant matrix elements using atomic wave functions, they found significantly better agreement between their theoretical line shapes and experimental data than could be achieved using the DOS alone. This approach was refined by Jennison ${ }^{23}$ who showed that the local charge at the site of the core would dominate the relative weighting for each angular momentum component.

The suggestion that Auger spectroscopy may provide a means of probing the local electronic structure of compound systems was made a number of years ago by Feibelman and coworkers. ${ }^{5}$ In that study, the lineshapes of several core-core-valence (CCV) Auger transitions on $\mathrm{GaAs}(110)$ were calculated. The CVV spectra were not addressed because they were considered to be experimentally difficult to access and because their lineshapes would require a self-fold of the local density of states. Cm the other hand, the CVV transitions have the considerable advantage that there is no core hole present in the final state and thus may be a better measure of the LDOS. As pointed out by Feibelman et al., ${ }^{5}$ the presence of the core hole has a large influence on the CCV Auger spectrum. In particular, a dramatic enhancement of the s-derived component of the DOS was seen in the calculated As- $M_{1} M_{4,5} V$ lineshape. These spectra were 
subsequently measured by Davis and Lagally ${ }^{24}$ and good overall agreement with the theory was found. In addition, differences between the As- and Ca- spectra supported the notion that Auger spectroscopy does probe the LDOS.

In a study of the CVV Auger lineshapes from GaAs(100) and ALAs(100), Svenssen et al. ${ }^{19}$ found good agreement between the SCDOS and their measured spectra. Here, the relative weightings of the different 1 -components was the same as was used in Fig. 3, however, they were unable to observe the sp- and ss-components of the spectrum and thus had no way to evaluate the validity of this choice. The sensitivity of their spectra to the local chemical environment was investigated by observing the Auger lineshape as the surface was prepared with various reconstructions associated with different $\mathrm{Ga}$ :As ratios. Only subtle changes were seen since only the pp-channel was measured.

Recently, there have been several attempts to isolate the surface contribution to CVV Auger spectra and understand these spectra in terms of the LDOS at the surface. ${ }^{11,25-27}$ In a discussion of $X$-ray excited Si $L_{2,3}$ VV Auger spectrum, Durbin and $\operatorname{Cog}^{26}$ reported a change in lineshape following deposition of a monolayer of $\mathrm{Ce}$ on the $\mathrm{Si}(111)$ surface. Their analysis indicated a reduction in the pp-contribution to the spectrum in the presence of the Ge overlayer and they argue that this is the result of the elimination of the surface contribution to the spectrum. They attribute the excess pp-contribution in the clean spectrum to increased p-charge associated with dangling bonds at the surface. These results have come under some scrutiny of late since the presence of the Ge overlayer changes the spectrum in a number of ways. ${ }^{27,28}$

The data we present in Fig. 2 shows that the relative intensity in the pp-region (ssregion) of the coincidence spectrum is dimirished (enhanced) compared to the singles. Experimentally, there are two possible origins of this effect. First, there may be emission not related to the decay of the $3 \mathrm{~d}$ core hole which is present in the singles spectrum; and second, the reduced electron sampling depth in coincidence is emphasizing the surface Auger spectrum. The first possibility is unlikely since there are no other constant kinetic energy emission features from GaAs which occur near $30 \mathrm{eV}$, and comparison of the coincidence data to a singles spectrum obtained at a higher photon energy show the same qualitative results. We therefore favor the second possibility and conclude that the apparent reduction in the pp-contribution to the Auger spectrum in coincidence reflects the electronic properties of the GaAs(110) surface.

The first possible source of differences between surface and bulk CVV lineshapes is the LDOS. This follows the approach used by Durbin and $\operatorname{Cog}^{26}$ for the Si(111) spectrum. For GaAs(110), however, we saw in Fig. 3 that the SCDOS are not significantly different from layer to layer other than a slight narrowing of the p-component at the surface. Furthermore, integration of the l-resolved DOS shows that the local charge of s-and p-character changes by only a few percent from one layer to the next. One might argue that the geometry of our surface 
differs than that used in the calculation. However, other work ${ }^{29}$ characterizing the surface electronic structure of GaAs(110) assuming a variety of surface relaxations has shown that other plausible geometries yield a smaller surface DOS of s-character than the geometry assumed here. Thus assuming other surface geometries would only make the comparison between theory and experiment worse. Therefore the arguments which were used ${ }^{26}$ to explain the Si data do not account for our results and we must go beyond this simple approximation for the Auger transition to understand the differences seen in the coincidence spectrum. We point out that hole-hole interaction in the final state is also an unlikely source of this discrepancy since there is not a significant narrowing of the valence band at the surface. In addition we would a redistribution to lower energy of spectral weight within the p-portion of the spectrum which is not observed.

The next aspect of the Auger transition to consider is the influence of the core hole. In a recent theoretical study of the CVV Auger spectra from simple metals, Almbladh and co workers 9,10 have shown that the initial core hole can strongly affect the relative contribution of each angular momentum component in the Auger lineshape. This is because the core hole lifetime is governed by the electronic configuration of the valence band in the presence of the core hole. In the case of GaAs, these ideas are consistent with the results of Lagally and Davis ${ }^{24}$ where their $M_{1} M_{4,5} \mathrm{~V}$ spectra indicate an enhanced s-component of the DOS for a transition which leaves a $3 d$ core hole in the final state. To explain our results for the As $\mathrm{M}_{4,5} \mathrm{VV}$ lineshape, we must invoke the further refinement that the presence of a $3 d$ core hole affects the surface electronic structure in a manner which is cistinct from that in the bulk. The discrepancy between our singles and coincidences a can be accounted for if the coupling of the $s-D O S$ ( $p$-DOS) at the surface were enhanced (reduced) compared to that in the bulk. Since there is no way to compare the singles and coincidence spectra on the same absolute scale, we cannot distinguish experimentally between these two possibilities. However, it is possible that the presence of an s-derived surface state, split off from the As s-levels into the heteropolar gap, ${ }^{21,} 29$ could be responsible for an increase in the coupling of the surface sderived LDOS in the presence of the core hole. We are currently calculating the electronic structure in this configuration.

Finally, we briefly discuss the low energy coincidence spectra. The Ga-M4,5V Auger spectrum is clearly observed in the coincidence spectrum of Fig. 4(a). Its kinetic energy and width are consistent with the pP-component of the SCDOS expected in a simple independent, electron mudel. The more interesting spectrum is the ss-component of the As- $\mathrm{M}_{4}, 5 \mathrm{VV}$ spectrum which is seem in Fig. 4(b). The SCDOS of Fig. 3(b) would indicate that this feature should occur about $22 \mathrm{eV}$ below threshold. In Fig. 2 we found that the threshold energy is about $32 \mathrm{eV}$ implying that the observed ss-feature lies about $24 \mathrm{eV}$ below threshold. This may be due to 
interaction of the two final state holes in the narrow As s- band. ${ }^{2}$ In addition, the $8 \mathrm{eV}$ peak of Fig. 4(b) has an amplitude of about 50 counts. To scale this spectrum to that of Fig. 2(a) we should multiply by a factor of about 3.5 which would equate the count rate at $13 \mathrm{eV}$. This means that the ss-peak at $8 \mathrm{eV}$ would have an intensity of about 175 counts on the scale of Fig. 2(a), giving is significant intensity compared to the pp-channel. This again indicates the unexpectedly large s-contribution to the As coincidence spectrum similar to what was seen at higher kinetic energies.

\section{Conclusions}

We have measured the Ga- and As- $\mathrm{M}_{4,5} \mathrm{VV}$ Auger spectra in coincidence with their respective core photoelectron lines. The coincidence technique enables us to separate the $\mathrm{Ga}$ and As contributions to the Auger spectrum and study their lineshapes independently. An unexpected reduction in the pp-component of the As Auger spectrum is attributed to a different response to the presence of a $3 d$ core hole at the surface and in the bulk. Only the pp-component of the Ga-M4,5V spectrum is observed and its lineshape is consistent with the independent electron approximation. The ss-component of the As-M $\mathrm{M}_{4,5} \mathrm{VV}$ spectrum is observed for the first time and appears to be at a lower energy and exhibit a larger relative intensity than would be expected from the independent electron approximation.

\section{Acknowledgements}

We would like to thank Prof. A Kahn for kindly providing the crystal used in this experiment and for many helpful discussions, and the staff of the National Synchrotron Light Source at Brookhaven National Laboratory supported by the Division of Materials Sciences of the Office of Basic Energy Sciences of the U.S. Department of Energy (DOE) under contracts Nos. DE-ACO2-76CH00016. We also thank Prof. E.W. Plummer for discussions and his assistance during the use of the U12 beamline funded by the NSF Materials Research Laboratory Program Grant No. DMR-85-19059. One of us (E.)) was funded by Analog Devices Career Development Grant and two of us (K.G. and R.A.B) were supported by the N.J Commission on Science and Technology under Grant No. 90-13. 


\section{References}

1. J.E. Houston and R.R. Rye. in Auger Electron Spectroscopy (eds. Briant, C.L., and R. P. Messmer) p. 65 (Academic Press, Inc., San Diego, 1988).

2. G.A. Sawatzky, ibid, p. 168

3. H.W. Haak, G.A. Sawatzki, and T.D. Thomas, Phys. Rev. Lett. 41, 1825 (1978).

4. H.W. Haak, G.A. Sawatzky, L. Ungier, J.K. Gimziewski, and T.D. Thomas, Rev. Sci. Instrum. 55, 696 (1984).

5. P. J. Feibelman, E. J. MCGuire and K. C. Pandey, Phys. Rev. B 16, 5499 (1977).

6. P. J. Feibelman, E. J. McGuire and K. C. Pandey, Phys. Rev, B 15, 2202 (1977).

7. P. J. Feibelman and E. J. MoGuire, Jhys. Rev. B 17,690 (1978).

8. E. Jensen, R. A. Bartynski, S. L. Hulbert, E. D. Johnson and R. Garrett, Phys. Rev. Lett. 62, 71 (1989).

9. C.-O. Almbladh and A. L. Morales, Phys. Rev, B 39, 3505 (1989),

10. C.-O. Almbladh, A. L. Morales, and G. Grossmann, Phys. Rev. B 39, 3489 (1989).

11. E. Jensen, R. A. Bartynski, M. Weinert, S. L. Hulbert, E. D. Johnson and R. F. Garrett, Phys. Rev. B 41, 12468 (1990).

12. E. Jensen, R.A. Bartynski, S.L. Hulbert, E.D. Johrson, (to be published).

13. Physical Electronics, Eden Prairie MN, model 255G,

14. B.P. Tonner, Nucl. Instrum. Methods 17, 133 (1980).

15. T. Maeda Wong, N.J. DiNardo, D. Heskett, and E.W. Plummer, Phys. Rev. B 41, 12342 (1990).

16. C.Y. Su, I. Lindau, P.W. Chye, P.R. Skeath, and W.E. Spicer, Phys. Rev. B 25, 4045 (1982).

17. M. Weinert, E. Wimmer and A.J. Freeman, Phys. Rev. B 26, 4571 (1982).

18. G.-X. Qian, R. M. Martin and D. J. Chadi, Phys. Rev. B 37, 1303 (1988).

19. S.P. Svensson, D.O. Nilsson, and T.G. Anderson, Phys. Rev. B 31, 5272 (1985).

20. T. Jarlborg and A. J. Freeman, Phys. Lett. 74A, 349 (1979).

21. J.R. Chelikowsky and M.L. Coheri; Phys. Rev. B 20, 4150 (1979).

22. D. E. Ramaker. in "Chemistry of Physics of Solids IV" (eds. Vanselow, R., and R. Howe) (Springer, Berlin 1982, 1982).

23. D. R. Jennison, Phys. Rev. Lett. 40, 807 (1978).

24. G.D. Davis and M.G. Lagally, J. Vac. Sci. Technol. 15, 1311 (1978).

25. J. C. Woid', B. B. Pate and P. Pianetta, Phys. Rev. B 39, 8539 (1989).

26. S. M. Durbin and T. Gog, Phys. Rev. Lett. 63, 1304 (1989).

27. V. Contiri, C. Presilla and F. Sacchetti, Phys. Rev. Lett. 64, 1844 (1990).

28. J.C. Woicik, P. Pianetta and T. Kendelewicz, Phys. Rev. B 40, 12463 (1989).

29. D.J. Chadi, Phys. Rev. B 18, 1800 (1978). 


\section{Figure Captions}

Figure 1: A wide scan of the photoemission spectrum from GaAs(110) excited with $90 \mathrm{eV}$ radiation. The dots above $70 \mathrm{eV}$ show the valence band enhanced by a factor of 200 . The dashed curve is the region of the As-M,, $\mathrm{VV}$ Auger spectrum enlarged by a factor of 5 . The $\mathrm{Ga}_{-} \mathrm{M}_{4,5} \mathrm{VV}$ Auger spectrum can be seen as a slight change in curvature of the secondary signal near $8 \mathrm{eV}$.

Figure 2: (a) The As-M4, $\mathrm{VVV}$ Auger spectrum obtained in coincidence with As-3d core photoelectrons. The open circles are the coincidence data, the clashed line is a polynomial furction which approximates the inelastic electron background. (b) The open circles are the coincidence data of panel (a) after background subtraction. The solid curve is the singles spectrum in the same region after subtraction of a similar background.

Figure 3: (a) Calculated layer resolved densittes of states at the As site for a 5-layer GaAs(110) slab resolved into their angular momentum components.

(b) The self-convolution of the DOS of panel (a) showing the pp-sp- and sscontributions.

Figure 4: (a) The Ga-M4,5V Auger spectrum from GaAs(110) obtained in coincidence with the $\mathrm{Ga}-3 \mathrm{~d}$ photoemission line. The circles in the upper panel are the coincidence data as acquired. The dashed line represents an approximate inelastic background. The lower panel shows the background subtracted data and the solid line is a digital smooth to these points.

(b)The ss-component of the As-M $4,5 \mathrm{VV}$ Auger spectrum obtained in coincidence with the As-3d Core line. The circles are the true coincidence data. The dashed line represents an approximate inelastic background. The lower spectrum is the background subtracted data (scaled $\times 2$ ) and the solid line is a digital smooth to these points. 


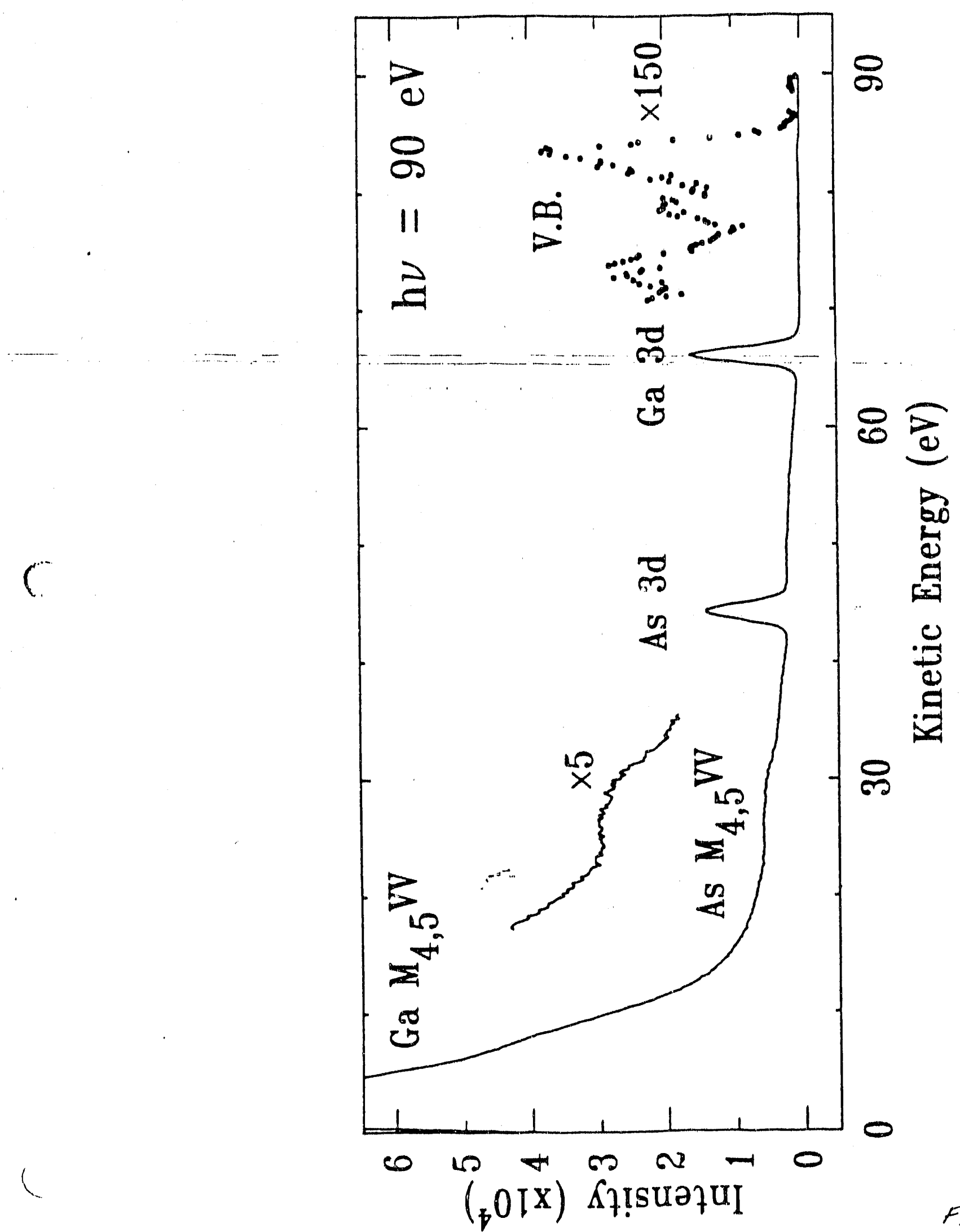

Fia. 1 


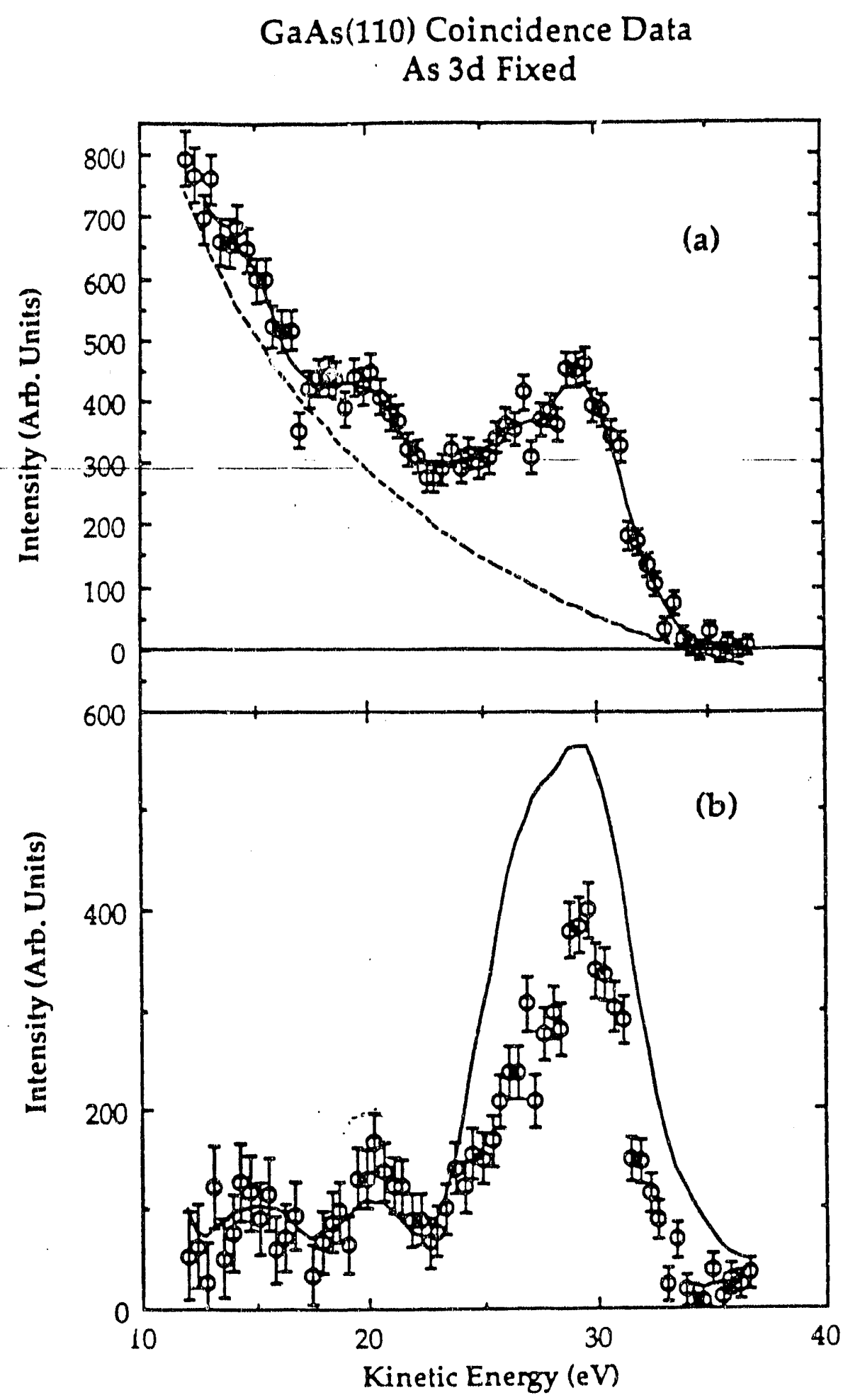

$F_{14} 2$ 
GaAs(110) DOS

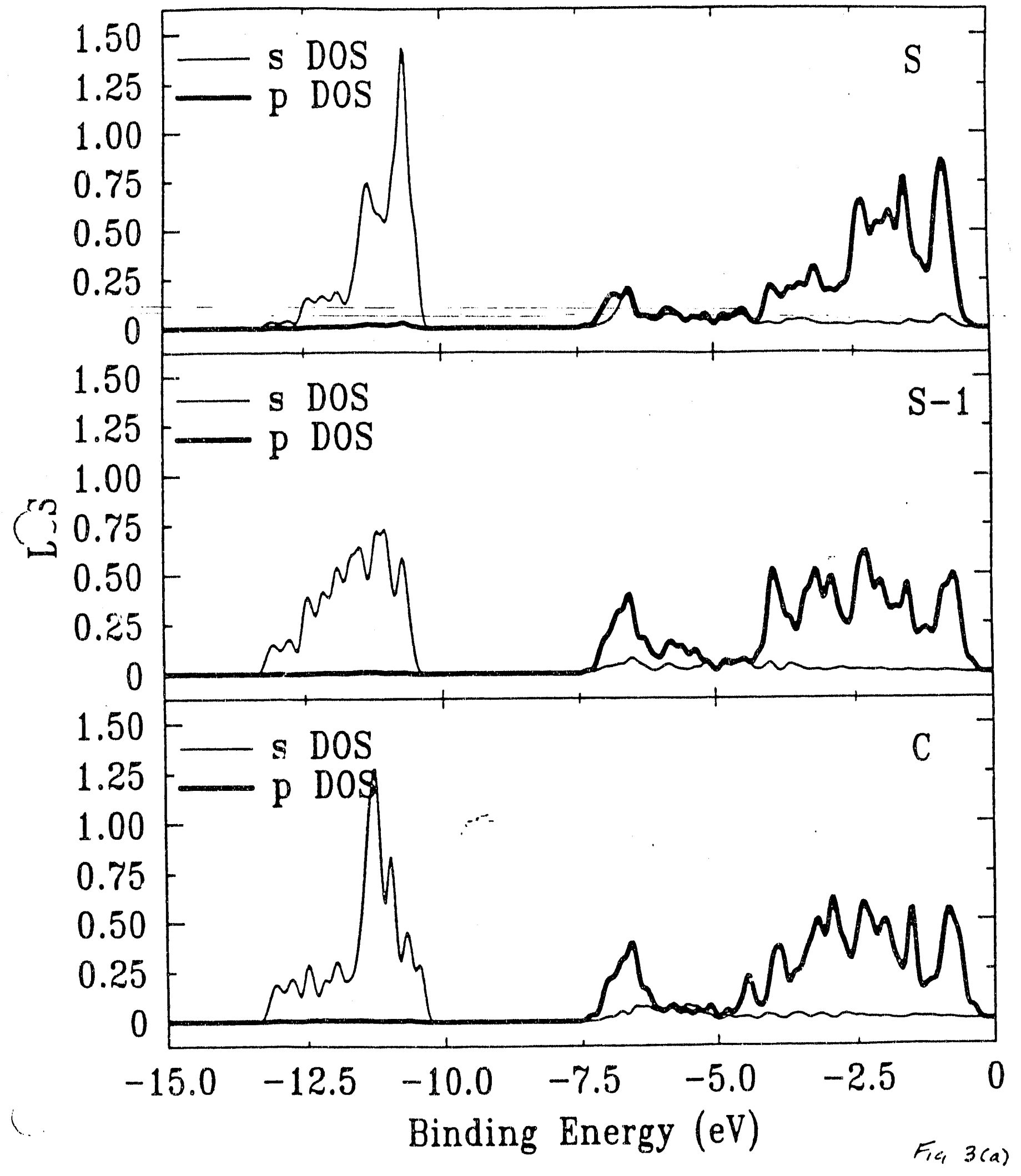


GaAs(110) SCDOS

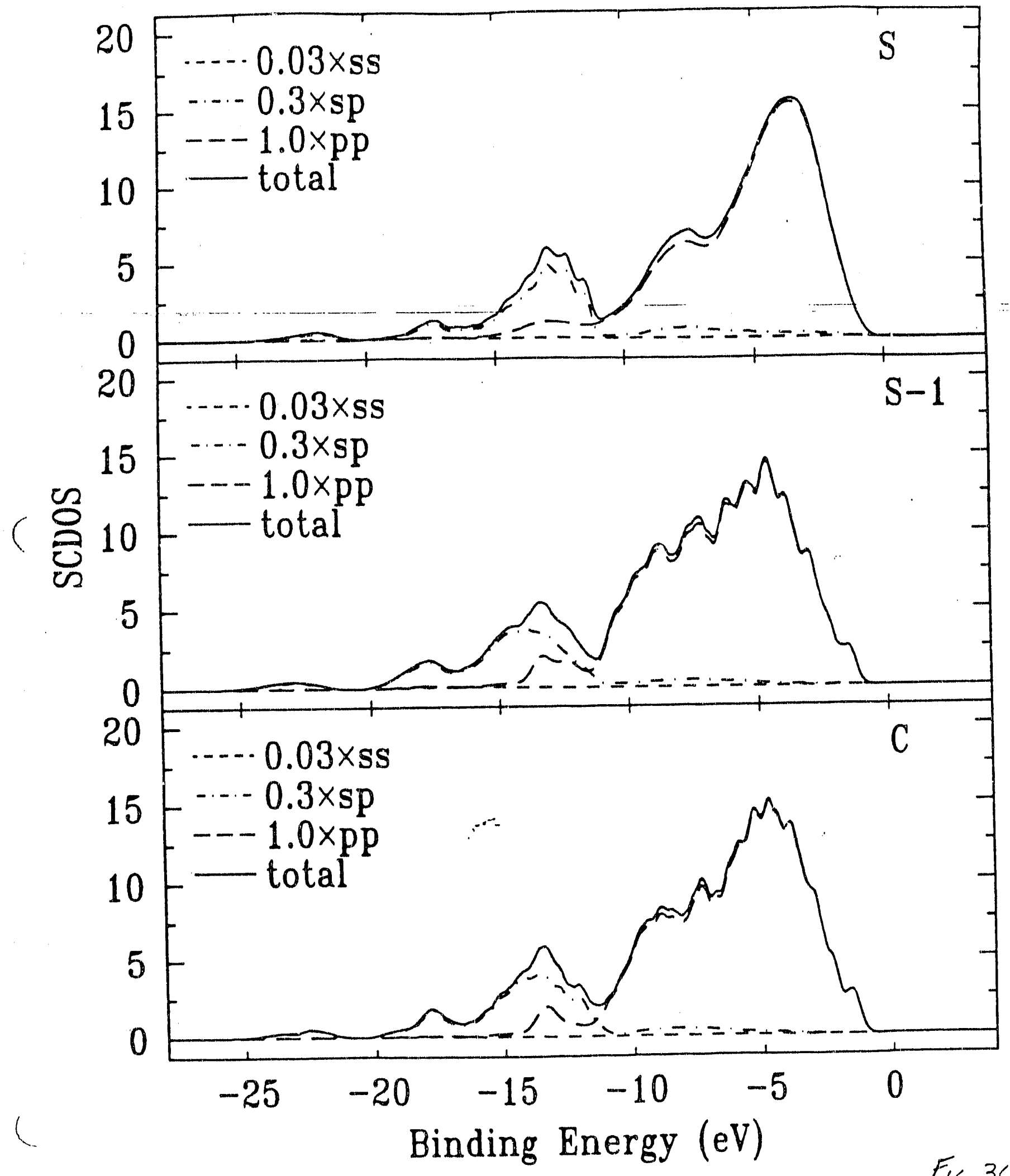

$F_{14} 3(6)$ 

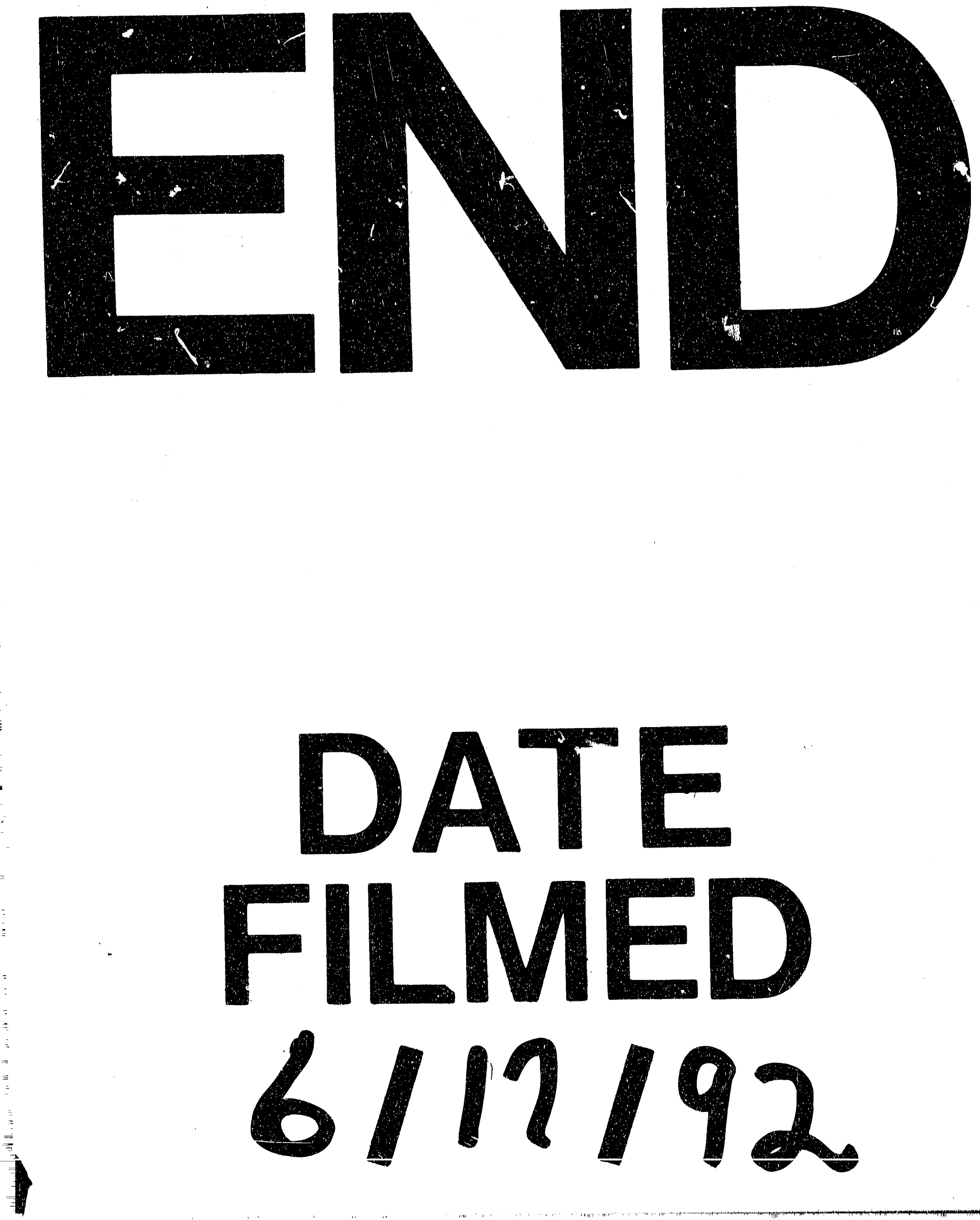
